\title{
Cáncer de próstata insignificante. Análisis de nuestra serie
}

\author{
Romero Selas E, Gómez Veiga F, Ponce Díaz-Reixa Jl, Pertega Díaz S*, González Martín M. \\ Servicio de Urología. *Servicio de Estadística. Hospital Juan Canalejo. La Coruña. \\ Actas Urol Esp. 2008;32(5):475-484
}

\section{RESUMEN}

CÁNCER DE PRÓSTATA INSIGNIFICANTE. ANÁLISIS DE NUESTRA SERIE

El objetivo de este estudio es el análisis de las piezas de prostatectomía radical de sujetos que a priori, en la biopsia parecen tener un cáncer de próstata insignificante. La relevancia de este tema está en la vigilancia expectante como posibilidad terapéutica para el cáncer de próstata, y que podría llevarse a cabo, en sujetos seleccionados si pudiésemos encontrar algoritmos diagnósticos para predecir los verdaderos tumores "insignificantes".

El grupo de pacientes seleccionados para el estudio son aquéllos con PSA menor o igual a 10, en los que la biopsia de próstata ecodirigida mostró uno solo de los 10 cilindros con tumor y un Gleason menor o igual a 6 . Este grupo de pacientes son considerados tumores potencialmente insignificantes, y nuestro objetivo era comprobar cuántos de ellos, realmente se comportaron como tal en la pieza de PR atendiendo a los criterios de insignificancia de: Gleason menor o igual a 6 y un volumen tumoral menor o igual al 5\% en la pieza de PR.

De los 394 pacientes con cáncer de próstata y criterios homogéneos para el estudio, seleccionamos los 53 que cumplían los requisitos de tumor potencialmente insignificante en la biopsia.

Nuestros resultados nos llevan a que sólo 22 de los 53 (41,5\%) pacientes tenían características de cáncer de próstata insignificante en la pieza de PR. Si bien es cierto, que el 92,5\% eran tumores órgano-confinados.

En conclusión, podemos decir, que estos tumores de bajo riesgo y baja carga tumoral en la biopsia, no siempre se corresponden con tumores insignificantes en la pieza de PR, aunque sí hemos observado que se tratará de tumores de bajo riesgo en la mayoría de los casos. Aún no existen algoritmos diagnósticos que nos permitan predecir aquéllos tumores susceptibles de un tratamiento con vigilancia expectante.
\end{abstract}

Palabras clave: Cáncer de próstata insifnificante: Vigilancia activa. Biopsia de próstata. Prostatectomía radical. PSA. Gleason.

\section{ABSTRACT}

INSIGNIFICANT PROSTATE CANCER. ANALYSIS OUR SERIES.

The objective of this study is to analyse the specimen of radical prostatectomy of patients who had, prior to the surgery insignificant prostate cancer biopsies. The end point is demonstrate the possibility of an active surveillance as a therapeutic option for prostate cancer in selected is the possibility of an active surveillance as therapeutic for the prostate cancer, in selected individuals if we are able to find diagnostic algorisms to predict the real insignificant tumours.

The selected group of patients for the study has a PSA less or equal 10, one positive core of prostatic transrectal ultrasound biopsy with a Gleason score less than 7. This group of patients is considerate as having a potential insignificant tumour. We will consider prostatectomy's specimens and the Gleason sore is less than 7 and the tumoral volume is les or equal to $5 \%$.

Of 394 patients with prostate cancer and homogeneous criterias for our study, we have selected 53 patients according to the criteria of insignificant tumour in the biopsy.

Our results showed that only 22 of 53 (41.5\%) patients were identified as having an insignificant prostate cancer in the RP specimens. Moreover $92.2 \%$ of this tumours were organ- confined.

In conclusion we are able to say that tumours of low- risk and low tumoral volume in the biopsy, do not correlate always to insignificant tumours in PR specimens, but we have observed that the majority are organ- confined tumours. Finally, the diagnostic's algorisms dies not to predict tumours that may safely treated with active monitoring. 
$\mathrm{E}$ s evidente que la era del PSA se ha instaurado por completo y que éste ha supuesto un gran avance en el diagnóstico más precoz del cáncer de próstata. Ante esto, nos encontramos con un incremento reciente del cáncer moderadamente diferenciado, de bajo volumen y potencialmente "insignificante".

Es bien conocido el manejo del cáncer de próstata localizado que pasa por la cirugía radical, radioterapia externa o braquiterapia. La decisión final entre las alternativas se basa fundamentalmente en una adecuada selección de los pacientes.

En la actualidad, el reto principal del urólogo ha cambiado y las nuevas expectativas rondan en torno a la posibilidad predecir el cáncer de próstata "insignificante" ya que existen corrientes que tratan de defender la posibilidad de un manejo con "vigilancia expectante". Existen distintos trabajos que describen sus series para defender sus teorías. Desde unos que creen que una cuidadosa selección de pacientes con cáncer de próstata de bajo riesgo son buenos candidatos para una vigilancia activa; y otros que abogan por la actitud terapéutica con fines curativos ya que no han podido demostrar una correlación exacta entre los resultados de la biopsia y los de la prostatectomía radical. El gran problema radica en encontrar el equilibrio en la balanza que nos permita no sobretratar a pacientes con tumores que podrían ser insignificantes, y tampoco dejar progresar aquellos que pareciendo insignificantes, no lo son realmente. La pregunta principal es si un bajo volumen tumoral en la biopsia, realmente traslada un bajo volumen en la pieza de prostatectomía radical.

Para ello, en este trabajo retrospectivo, analizamos los resultados patológicos de la pieza de prostatectomía radical en pacientes de bajo riesgo, con mínima enfermedad en la biopsia.

\section{MATERIAL Y MÉTODOS}

Entre Julio de 1999 y Septiembre de 2005 en el CHU Juan Canalejo se han sometido a una prostatectomía radical, con biopsia prostática previa de 10 cilindros un total de 473 pacientes con cáncer de próstata. Descartamos aquéllos con RTU previa, bloqueo hormonal, diagnóstico sin biopsia previa, diagnóstico en sucesivas biopsias o con biopsias de saturación. La biopsia que realizamos en todos ellos es endorrectal, con seguimiento ecográfico y de 10 cilindros. Finalmente, analizamos sólo los pacientes que disponían además de valores de PSA y sus fracciones previo a la biopsia, Gleason de la biopsia y número de cilindros positivos en ésta.

Por lo tanto, para homogeneizar la muestra, incluimos en el estudio los 394 pacientes que cumplían los requisitos y de los que conocíamos todos sus datos.

Los parámetros pre-biopsia estudiados son; el PSA sérico y sus fracciones, el gleason de la biopsia, el número de cilindros positivos y el estadio clínico. Todos los pacientes fueron sometidos a prostatectomía radical y los parámetros postbiopsia analizados son el Gleason, el volumen tumoral y el estadio patológico.

La edad media de los pacientes es de 66 años. El PSA medio es de 8,57 ng/dl, y el volumen prostático medio de 51,61 cc. En la tabla que se presenta a continuación, se exponen las características de los pacientes incluidos en la revisión (Tabla 1).

Para nuestro trabajo, definimos los dos conceptos que vamos a manejar:

Como Cáncer de próstata potencialmente insignificante en la biopsia, aquéllos casos que cumplen los siguientes requisitos:

Un sólo cilindro positivo en la biopsia (BPE de 10 cilindros).

Gleason $\leq 6$.

$\mathrm{PSA} \leq 10$.

Como Cáncer de próstata insignificante tras Prostatectomía Radical aquéllos que finalmente cumplen los siguientes requisitos:

Volumen tumoral $\leq 5 \%$.

Gleason $\leq 6$.

El estudio se centra en el grupo de pacientes con características de tumor potencialmente insignificante por reunir las características antes descritas para ello. 53 de los 394 iniciales (12,7\%) los englobamos en el grupo de cáncer de próstata insignificante, es decir, con PSA $<10$, gleason en la biopsia $<7$ y un solo cilindro positivo. En la siguiente tabla se presentan el total de los pacientes del estudio divididos en grupos según dichos parámetros (Tabla 2).

Revisamos las características del grupo de pacientes con cáncer de próstata insignificante en la biopsia, y se exponen en la siguiente tabla en comparación con los del grupo de tumores no insignificantes en la biopsia. La media de Psa en nuestro grupo de estudio es de 6,06, 81,10\% tienen un tacto 
Tabla 1. Características generales de la serie de pacientes estudiados.

\begin{tabular}{|c|c|c|c|c|c|c|}
\hline & & Media & DT & Mediana & $\mathbf{n}$ & $\%$ \\
\hline \multicolumn{2}{|l|}{ Edad } & 66,06 & 6,23 & 68,00 & & \\
\hline \multirow[t]{2}{*}{ Tacto rectal } & Normal & & & & 259 & 66,1 \\
\hline & Anormal & & & & 133 & 33,9 \\
\hline \multirow[t]{3}{*}{ PSA } & & 8,57 & 10,25 & 6,90 & & \\
\hline & $<10$ & & & & 305 & 77,4 \\
\hline & $\geq 10$ & & & & 89 & 22,6 \\
\hline \multicolumn{2}{|l|}{ Volumen prostático } & 51,61 & 25,58 & 45,00 & & \\
\hline \multirow[t]{3}{*}{ Gleason de biopsia } & $2-6$ & & & & 257 & 65,2 \\
\hline & 7 & & & & 124 & 31,5 \\
\hline & 8-10 & & & & 13 & 3,3 \\
\hline \multirow[t]{3}{*}{ Gleason tras prostatectomia } & $2-6$ & & & & 140 & 35,5 \\
\hline & 7 & & & & 197 & 50,0 \\
\hline & 8-10 & & & & 57 & 14,5 \\
\hline \multirow[t]{2}{*}{$\mathrm{N}^{\circ}$ de cilindros positivos } & 1 cilindro positivo & & & & 89 & 22,6 \\
\hline & $>1$ cilindro positivo & & & & 305 & 77,4 \\
\hline \multicolumn{2}{|l|}{$\%$ de cilindros positivos } & 29,42 & 17,66 & 20,00 & & \\
\hline \multirow[t]{3}{*}{ Estadío clínico } & cT1 & & & & 198 & 50,9 \\
\hline & сT2 & & & & 187 & 48,1 \\
\hline & ст3 & & & & 4 & 1,0 \\
\hline Estadío patológico & To & & & & 1 & ,3 \\
\hline \multicolumn{2}{|l|}{ Tumor confinado a la próstata } & & & 300 & 76,1 & \\
\hline \multicolumn{2}{|l|}{$\begin{array}{l}\text { Tumor extracapsular sin } \\
\text { afectar vesículas ni ganglios }\end{array}$} & & & 67 & 17,0 & \\
\hline \multicolumn{2}{|c|}{$\begin{array}{l}\text { Afectación de vesículas seminales } \\
\text { sin afectación de nodos }\end{array}$} & & & 25 & 6,3 & \\
\hline \multicolumn{2}{|l|}{ Afectación de ganglios } & & & 1 & ,3 & \\
\hline
\end{tabular}

rectal normal, $73 \%$ son un estadio clínico cT1, $26,9 \%$ cT2 y ninguno cT3. (Tabla 3 ).

\section{RESULTADOS}

Los parámetros evaluados tras haber realizado la prostatectomía radical en el grupo de pacientes con cáncer insignificante en la biopsia son el volumen tumoral y el Gleason en la pieza de PR. Comparamos estos dos parámetros respecto a cada uno de los analizados previamente, y obtenemos el grupo

Tabla 2. Distribución de pacientes según PSA, Gleason y $\mathrm{n}^{\circ}$ cilindros+.

\begin{tabular}{|c|c|c|c|c|}
\hline \multirow[b]{2}{*}{ PSA total } & \multicolumn{3}{|c|}{$\begin{array}{l}\mathbf{N}^{\circ} \text { de cilindros } \\
\text { positivos }\end{array}$} & \multirow[b]{2}{*}{ Total } \\
\hline & $\begin{array}{c}\text { Gleason } \\
\text { de biopsia }\end{array}$ & $\begin{array}{c}1 \text { cilindro } \\
\text { positivo }\end{array}$ & $\begin{array}{l}>1 \text { cilindro } \\
\text { positivo }\end{array}$ & \\
\hline \multirow[t]{2}{*}{$<10$} & $<7$ & 53 & 140 & 193 \\
\hline & $\geq 7$ & 9 & 103 & 112 \\
\hline \multirow[t]{2}{*}{$\geq 10$} & $<7$ & 24 & 40 & 64 \\
\hline & $\geq 7$ & 3 & 22 & 25 \\
\hline
\end{tabular}

de pacientes que aunando ambos requisitos, consideramos como cáncer de próstata insignificante.

En las siguientes tablas se muestra la relación entre los hallazgos de la prostatectomía y los datos clínicos tras la biopsia.

Tablas que evalúan el volumen tumoral en la pieza de PR respecto al Gleason en la biopsia $(<7$ o $\geq 7)$, el Psa previo $(<10$ o $\geq 10)$ y el número de cilindros positivos en la biopsia (1 o más de 1): (Tablas 4-6).

Tablas que evalúan el Gleason tras la PR respecto al número de cilindros positivos, el Psa y el Gleason en la biopsia (Tablas 7-9).

Al analizar la pieza de prostatectomía radical, nos encontramos con un 1,9\% (1 paciente) de pT0, y un $92,5 \%$ (49) de tumores eran confinados a la próstata. Sólo $3,8 \%{ }^{2}$ eran tumores extracapsulares sin afectación de vesículas ni adenopatías, 1,9\%1 con afectación de vesículas aunque sin ganglios, y ninguno de los pacientes presentaban afectación de 
Tabla 3. Características de pacientes con tumores insignificantes y no insignificantes.

\begin{tabular}{|c|c|c|c|c|c|c|c|c|c|}
\hline & & & \multicolumn{3}{|c|}{$\begin{array}{l}\text { Tumor no insignificante } \\
(>1 \text { cilindro biopsia, } \\
\text { Gleason }>6 \text { o PSA }>10 \text { ) }\end{array}$} & \multicolumn{3}{|c|}{$\begin{array}{c}\text { Tumor insignificante } \\
(1 \text { cilindro biopsia, } \\
\text { Gleason } \leq 6, P S A \leq 10)\end{array}$} & \multirow[b]{2}{*}{$\mathbf{p}$} \\
\hline & & & Media & $\mathbf{n}$ & $\%$ & Media & $\mathbf{n}$ & $\%$ & \\
\hline Edad & & & 65,88 & & & 67,21 & & & 0,151 \\
\hline \multirow[t]{2}{*}{ Tacto rectal } & normal & & & 216 & 63,70 & & 43 & 81,10 & 0,013 \\
\hline & anormal & & & 123 & 36,30 & & 10 & 18,90 & \\
\hline \multirow{2}{*}{\multicolumn{2}{|c|}{$\begin{array}{l}\text { PSA } \\
\text { Volumen prostático }\end{array}$}} & & 8,96 & & & 6,06 & & & 0,003 \\
\hline & & & 50,47 & & & 58,75 & & & 0,077 \\
\hline \multirow[t]{3}{*}{$\begin{array}{l}\text { Gleason } \\
\text { biopsia }\end{array}$} & 2 a 6 & & & 204 & 59,80 & & 53 & 100,00 & - \\
\hline & & 7 & & 124 & 36,40 & & 0 & 0,00 & \\
\hline & 8 a 10 & & & 13 & 3,80 & & 0 & 0,00 & \\
\hline \multirow[t]{3}{*}{ Gleason PR } & 2 a 6 & & & 102 & 29,90 & & 38 & 71,70 & $<0,001$ \\
\hline & & 7 & & 185 & 54,30 & & 12 & 22,60 & \\
\hline & 2 a 6 & & & 54 & 15,80 & & 3 & 5,70 & \\
\hline \multirow{4}{*}{$\begin{array}{l}\text { Estadio } \\
\text { clínico }\end{array}$} & cT1 & & & & & & & & \\
\hline & & & & 160 & 47,50 & & 38 & 73,10 & 0,001 \\
\hline & cT2 & & & 173 & 51,30 & & 14 & 26,90 & \\
\hline & сT3 & & & 4 & 1,20 & & 0 & 0,00 & \\
\hline \multicolumn{2}{|c|}{$\begin{array}{l}\begin{array}{l}\text { Estadio } \\
\text { patológico }\end{array} \\
\text { pTO }\end{array}$} & & & 0 & 0,00 & & 1 & 1,90 & 0,001 \\
\hline \multicolumn{2}{|c|}{$\begin{array}{l}\text { Tumor confinado } \\
\text { a la próstata }\end{array}$} & & & & 251 & $73,60 \%$ & & 49 & $92,50 \%$ \\
\hline \multicolumn{2}{|c|}{$\begin{array}{l}\text { Tu. extracapsular } \\
\text { sin vvss ni ganglios }\end{array}$} & & & & 65 & $19,10 \%$ & & 2 & $3,80 \%$ \\
\hline \multicolumn{2}{|c|}{$\begin{array}{l}\text { Afectación de vvss } \\
\text { sin ganglios }\end{array}$} & & & & 24 & $7,00 \%$ & & 1 & $1,90 \%$ \\
\hline \multicolumn{2}{|l|}{$\begin{array}{l}\text { Afectación } \\
\text { de ganglios }\end{array}$} & & & & 1 & $0,30 \%$ & & 0 & $0,00 \%$ \\
\hline
\end{tabular}

ganglios. Por lo tanto, la mayoría eran tumores confinados a la próstata.

También se ve en las tablas que 38 pacientes de esos 53 con cáncer potencialmente insignificante en la biopsia tienten un Gleason $<7$ en la pieza de PR, y 25 de ese grupo estudiado tienen un volumen tumoral $<5 \%$ en la pieza. Si unimos los dos parámetros de estudio en la pieza, obtenemos el grupo de pacientes que finalmente nos interesa y que se corresponde con el verdadero grupo con cáncer de próstata insignificante, según los criterios definidos al inicio. Un total de 22 pacientes de los 53 con cáncer insignificante en la biopsia, son verdaderamente insignificantes en la pieza de PR y representan un $41,5 \%$.

Otro de los resultados observados es que pacientes con volúmenes tumorales $<5 \%$, se corresponden con mayores volúmenes prostáticos. Para esta explicación se exponen la siguiente Tabla 12 y Fig. 1.
Resumimos estos resultados en los siguientes puntos:

- No todos los pacientes con tumores potencialmente "insignificantes", se comportaron como tal.

- De los 53 con PSA< 10, gleason < 7 y un solo cilindro + en la biopsia, solo 25 (47\%) presentaron un volumen tumoral $<5 \%$.

- Si le añadimos factores en contra de enfermedad "insignificante", este\% va disminuyendo: si $>1$ cilindro (31\%), si gleason $>7(20 \%)$, si PSA $>10$ (13\%).

- Sólo 38 de estos 53 (72\%) presentaron un Gleason $<6$.

- Sin embargo, si añadimos mas de un cilindro +, los $\%$ de Gleason son 47\%, 49\% y 3\% para los gleason de $\mathrm{PR}<6,7$, y 8-10 respectivamente.

- Sólo un 6\% eran tumores "no órgano-confinados": $4 \%$ extracapsular y $2 \%$ afectación de vvss. Ninguno con adenopatías positivas. 
Tabla 4. Volumen tumoral final en relación con el Gleason de la biopsia.

\begin{tabular}{lccc}
\hline Gleason de biopsia & \multicolumn{3}{c}{ Volumen tumoral } \\
\hline & $>\mathbf{5 \%}$ & $<\mathbf{5 \%}$ & Total \\
$<\mathbf{7}$ & 168 & $\mathbf{8 9}$ & 257 \\
$\geq \mathbf{7}$ & 110 & 27 & 137 \\
Total & 278 & 116 & $\mathbf{3 9 4}$ \\
\hline
\end{tabular}

Chi-cuadrado: $\mathrm{p}=0,002$

Tabla 5. Volumen tumoral final en relación con el PSA previo.

\begin{tabular}{lccc}
\hline PSA & \multicolumn{3}{c}{ Volumen tumoral } \\
\hline & $>\mathbf{5 \%}$ & $<\mathbf{5 \%}$ & Total \\
$<\mathbf{1 0}$ & 214 & $\mathbf{9 1}$ & 305 \\
$\geq \mathbf{1 0}$ & 64 & 25 & 89 \\
Total & 278 & 116 & $\mathbf{3 9 4}$ \\
\hline
\end{tabular}

Chi-cuadrado: $\mathrm{p}=0,750$

Tabla 6. Volumen tumoral final en relación con el número de cilindros positivos en la biopsia.

\begin{tabular}{lccc}
\hline $\mathbf{N}^{\mathbf{0}}$ cilindros positivos & \multicolumn{3}{c}{ Volumen tumoral } \\
\hline & $>\mathbf{5 \%}$ & $\mathbf{<} \%$ & Total \\
$\mathbf{1}$ & 50 & $\mathbf{3 9}$ & 89 \\
$>\mathbf{1}$ & 228 & 77 & 305 \\
Total & 278 & 116 & $\mathbf{3 9 4}$ \\
\hline
\end{tabular}

Chi-cuadrado: $p<0,001$

Tabla 7. Gleason tras la prostatectomía en relación con el número de cilindros positivos en la biopsia.

\begin{tabular}{lccc}
\hline $\mathbf{N}^{\mathbf{0}}$ cilindros positivos & \multicolumn{3}{c}{ Gleason tras } \\
& prostatectomia & \\
\hline & $<\mathbf{7}$ & $\geq \mathbf{7}$ & Total \\
$\mathbf{1}$ & $\mathbf{5 3}$ & 36 & 89 \\
$>\mathbf{1}$ & 87 & 218 & 305 \\
Total & 140 & 254 & $\mathbf{3 9 4}$ \\
\hline
\end{tabular}

Chi cuadrado: $\mathrm{p}<0,001$

Tabla 8. Gleason tras la prostatectomía en relación con el valor de PSA total.

\begin{tabular}{lccc}
\hline PSA & \multicolumn{3}{c}{$\begin{array}{c}\text { Gleason tras } \\
\text { prostatectomia }\end{array}$} \\
\hline & $<\mathbf{7}$ & $\mathbf{2 7}$ & Total \\
$<\mathbf{1 0}$ & $\mathbf{1 1 0}$ & 195 & 305 \\
$\geq \mathbf{1 0}$ & 30 & 59 & 89 \\
Total & 140 & 254 & $\mathbf{3 9 4}$ \\
\hline
\end{tabular}

Chi-cuadrado: $\mathrm{p}=0,683$

Tabla 9. Gleason tras la prostatectomía en relación con el gleason de la biopsia.

\begin{tabular}{lccc}
\hline Gleason de biopsia & \multicolumn{3}{c}{$\begin{array}{c}\text { Gleason tras } \\
\text { prostatectomia }\end{array}$} \\
\hline & $<\mathbf{7}$ & $\geq \mathbf{7}$ & Total \\
$\mathbf{2 7}$ & $\mathbf{1 3 1}$ & 126 & 257 \\
$\geq \mathbf{7}$ & 9 & 128 & 137 \\
\hline Total & 140 & 254 & $\mathbf{3 9 4}$ \\
\hline
\end{tabular}

Chi-cuadrado: $\mathrm{p}<0,001$
Tabla 10. Gleason tras la prostatectomía en relación a los resultados de la biopsia en cada paciente se obtienen los siguientes datos:

\begin{tabular}{lccc}
\hline Resultados clínicos & \multicolumn{3}{c}{$\begin{array}{c}\text { Gleason tras } \\
\text { prostatectomía }\end{array}$} \\
\hline & $<7$ & $\geq 7$ & Total \\
$\begin{array}{l}\text { Más de } 1 \text { cilindro positivo, } \\
\text { Gleason biopsia >6 o PSA }>\mathbf{1 0}\end{array}$ & 102 & 239 & 341 \\
$\begin{array}{l}\text { 1 cilindro positivo, Gleason } \\
\text { biopsia } \leq 6, \text { PSA } \leq \mathbf{1 0}\end{array}$ & $\mathbf{3 8}$ & 15 & 53 \\
Total & 140 & 254 & $\mathbf{3 9 4}$ \\
\hline
\end{tabular}

Chi-cuadrado; $\mathrm{p}<0,001$

Tabla 11. Volumen tumoral según los hallazgos en la biopsia.

\begin{tabular}{lccc}
\hline Resultados de la biopsia & \multicolumn{3}{c}{ Volumen tumoral } \\
\hline $\begin{array}{l}\text { Más de } 1 \text { cilindro positivo, } \\
\text { Gleason biopsia }>\mathbf{6} \text { ó PSA }>\mathbf{1 0}\end{array}$ & 250 & 91 & 341 \\
$\begin{array}{l}\mathbf{1} \text { cilindro positivo, Gleason } \\
\text { biopsia } \leq 6, \text { PSA } \leq \mathbf{1 0}\end{array}$ & 28 & 25 & Total \\
Total & 278 & 116 & $\mathbf{3 9 4}$ \\
\hline
\end{tabular}

Chi-cuadrado: $\mathrm{p}=0,002$

- Un 41'5\% de los 53 iniciales resultaron gleason $<7$ y volumen $<5 \%$ tras la PR y por lo tanto, tumores insignificantes.

\section{DISCUSIÓN}

El uso del PSA (prostate specific antigen) asociado con el uso extendido de la biopsia transrectal ecodirigida en los últimos años ha dado lugar a un cambio en las características del cáncer de próstata. Esto se manifiesta con un aumento del diagnóstico de tumores moderadamente diferenciados, con bajo volumen tumoral y por lo tanto, potencialmente insignificantes. Además, un volumen mayor de pacientes son candidatos a terapias curativas por tener tumores localizados.

Igualmente, y como también se aprecia en nuestro trabajo, el diagnóstico de tumores de alto grado con PSA bajo, incluso por debajo de 4, como demuestran muchos trabajos, es significativo, con lo que podemos aseverar que uno de los grandes dilemas sigue siendo encontrar el límite de normalidad del PSA, ya que no podemos decir todavía que exista un punto de corte de PSA con alta sensibilidad y especificidad simultáneamente para monitorizar la salud del varón respecto al cáncer 
Tabla 12

\begin{tabular}{lccccccc}
\hline Volumen tumoral & Media & N & Desv. típ. & Mediana & Mínimo & Máximo & p \\
\hline$>5 \%$ & 47,59 & 245 & 22,63 & 42 & 1 & 150 & $<0,001$ \\
$<5 \%$ & 61,18 & 103 & 29,50 & 58 & 3 & 166 & 166 \\
Total & 51,61 & 348 & 25,58 & 45 & 1 & \\
\hline
\end{tabular}

Estos datos se representan en la Figura 1.

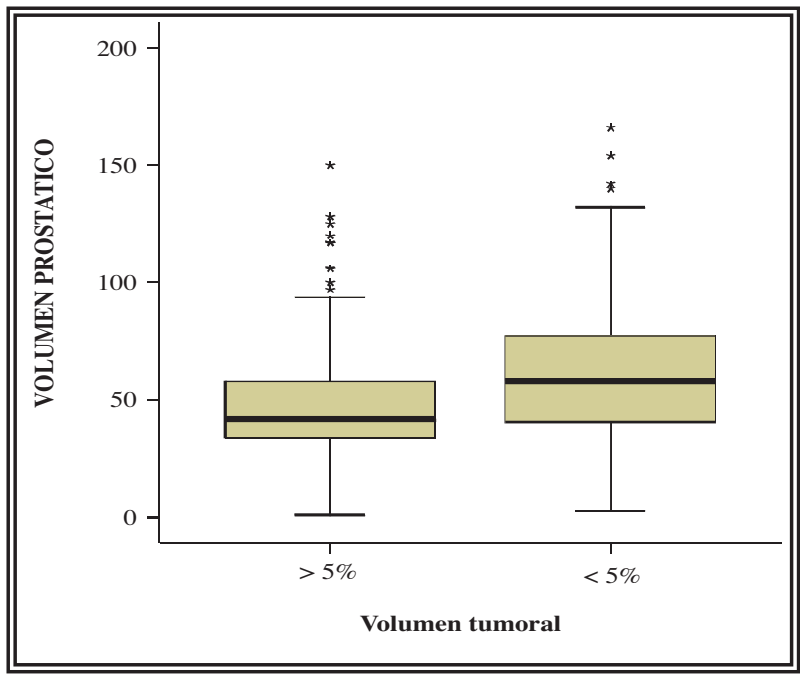

FIGURA 1

de próstata ${ }^{1,2}$. Una solución a este problema ha sido el descubrimiento y uso de las fracciones de PSA y su ayuda en el diagnóstico de cáncer de próstata para restringir un poco más el número de biopsias en pacientes con valores de PSA en el rango de $2-5$ y $4^{3}$.

A consecuencia de la extensión de los programas de screening y diagnóstico precoz de cáncer de próstata, se están realizando biopsias prostáticas con niveles bajos de PSA (por encima de 2’5 $\mathrm{ng} / \mathrm{ml}$ ), lo que lleva a una detección de tumores en estadios muy precoces, que podrían resultar insignificantes a lo largo de la vida de esos pacientes. Es este sobre-diagnóstico, el que ha dado lugar a la aparición del concepto de tratamiento expectante con intención curativa ya que se nos plantea la posibilidad de estar "sobretratando" a un número de pacientes considerable, si tenemos en cuenta que muchos de estos tumores diagnosticados nunca llegarían a ser clínicamente significativos ni dar problemas al paciente a lo largo de su vida. Sin embargo, algunos trabajos postulan que aquellos hombres diagnosticados de cáncer de próstata viven más que aquéllos que no se diagnostican ${ }^{4-7}$.
En base a esto, numerosos grupos han intentado establecer aquellas variables que podrían comportarse como factores pronósticos y que más correlación guardan con el grado y extensión del tumor para poder predecir el tipo de tumor final y dar el tratamiento más adecuado a cada paciente ${ }^{8}$.

El cáncer de próstata localizado ha demostrado una gran heterogeneidad en su historia natural. En la literatura se dice que está presente histológicamente en un 30\% de los varones en torno a 50 años; se diagnostica en torno a un 16\% y el riesgo de muerte por cáncer de próstata es menor al 4\%. Por todo ello, con el fin de no tratar a los tumores potencialmente insignificantes se ha considerado el manejo con tratamiento expectante o vigilancia activa de esta enfermedad, ya que existen autores que defienden esta terapéutica basándose en que en muchos casos estamos sobretratando a un paciente que con un cancer de próstata de bajo riesgo y un baja probbilidad de morir por esta causa. Estos defienden la vigilancia activa basada en un seguimiento exhaustivo del paciente con monitorización del PSA, haciendo especial atención al tiempo de doblaje del mismo, sumado a una buena comunicación con el paciente, que debe conocer bien su problema y ser capaz de vivir sin la ansiedad de un "cancer no tratado"9-11.

Desde que Epstein en 1994 definió por primera vez el concepto de cáncer de próstata "insignificante" basándose en hallazgos de piezas de prostatectomía radical hasta hoy, este concepto sigue en discusión y no podemos decir que existan parámetros totalmente seguros. Sus criterios incluian: densidad de PSA $<0,15 \mathrm{ng} / \mathrm{ml}$, Gleason $\leq 6$, tumor en menos de 3 de los 6 cilindros de la biopsia, y la presencia de $<$ del 50\% de volumen tumoral en cada uno de ellos. Estudió el grupo de cáncer de próstata T1c y concluyó que es un grupo de pacientes heterogéneo, que un $84 \%$ de los pacientes presentaron tumores significantes y solo un 16\% fueron considerados insignificantes; que el PSA, la densidad de PSA y los 
hallazgos patológicos de la biopsia por punción eran predictores seguros de la extensión del tumor por lo que este grupo de pacientes con tumores insignificantes eran buenos candidatos para el seguimiento con determinaciones de PSA seriadas y biopsias repetidas.

En otros muchos trabajos se ha demostrado que el porcentaje de cilindros positivos en la biopsia y la cantidad de volumen tumoral son los factores predictivos con mayor peso en el análisis preoperatorio de los pacientes con cáncer de próstata. Otros, sin embargo, concluyen que el volumen tumoral en la biopsia no puede predecir la cantidad de cáncer en la PR.

Por eso, la gran pregunta sigue siendo: ¿en qué tipo de tumores, con que criterios y en qué porcentaje podemos considerar que un tumor va a ser como nos lo define la biopsia diagnóstica? Y sobretodo, ¿en cuáles de los que a priori parecen insignificantes, esto se mostrará así en la pieza de prostatatectomía radical?

En estudios más recientes se concluye que los hallazgos clínico-patológicos favorables en pacientes con cáncer insignificante, comparado con los de los significantes, y la combinación del Gleason en la biopsia $<7$ y el porcentaje de cilindros positivos en ésta, podrían ser usados como predictores de enfermedad insignificante ${ }^{12,13}$.

En base a esto, algunos autores han desarrollado nomogramas para predecir aquellos tumores que podrian ser insignificantes basándose en variables pre-tratamiento (estadio clínico, grado de Gleason, PSA y el volumen tumoral en la biopsia). Avalan la teoría de que podrían beneficiar al paciente y al clínico para tomar una decisión ante varias opciones de tratamiento ${ }^{14,15}$. Son bien conocidos los criterios de Epstein para predecir el cáncer de próstata clínicamente insignificante; recientemente han sido evaluados para ver su correspondencia en hombres europeos con cáncer de próstata y han concluido que infravaloran en un $24 \%$ la naturaleza de esta enfermedad en este grupo de hombres y que es preciso prestar atención a este tipo de tumores, si nos basamos únicamente en dichos criterios ${ }^{16}$.

Otros autores concluyen tras estudiar las piezas de prostatectomía radical para ver la correlación patológica entre éstas y las biopsias de 6 cilindros, que no existe una correlación exacta entre ambas; y que la combinación de 1 solo cilindro positivo en la biopsia, de menos de $3 \mathrm{~mm}$ y que no contenga grado de Gleason 4 ni 5 es probablemente el mejor predictor de cáncer de próstata de menos de 0.5 cc en hombres con tumores no palpables. También concluyen, que en solo un 36\% de los cánceres de próstata, el grado tumoral en la biopsia se corresponde con el de la pieza de prostatectomía radical. Y sólo en $3 \%$ de las biopsias con $3 \mathrm{~mm}$ de tumor, el volumen tumoral de la pieza fue menor a $0,5 \%{ }^{17}$.

El cáncer de próstata en la pieza de prostatectomía radical es considerado "insignificante" si cumple los requisitos de bajo volumen tumoral $(<0,5 \mathrm{ml})$ y un grado de diferenciación bajo-moderado (ningún elemento de Gleason grado 4).

La frecuencia de cáncer de próstata insignificante en piezas de prostatectomía radical de T1c es controvertida. Usando un volumen tumoral menor a 0,5 cc, sin componentes de alto grado tumoral como definición de tumor insignificante, las distintas series definen un porcentaje de insignificantes entre 16 y 83\%. Usando criterios más restrictivos para cáncer de próstata insignificante (volumen tumoral $<0,5 \mathrm{cc}$, órgano-confinado y grado de Gleason de 2 a 4), Epstein y Gadner clasificaron sólo un 6\% de los T1c como cánceres insignificantes ${ }^{18,19}$.

Teniendo en cuenta esto, muchos autores y grupos de trabajo han realizado estudios de comparación entre las características del tumor en la biopsia y en la pieza de prostatectomía radical. Incluso hay grupos que han ido más allá y han estudiado la supervivencia del grupo con tratamiento y el de la observación en el cáncer de próstata en varones de edad avanzada, y han concluido que la supervivencia es mayor en el grupo de tratamiento, pero engloban no sólo pacientes con bajo riesgo, sino también, aquéllos con riesgo intermedio. También concluyen, que estos resultados deberían ser validados con estudios randomizados ${ }^{20}$.

El grupo The Johns Hopkins Hospital presenta en su serie de tumores potencialmente insignificantes, una correspondencia del $83 \%$ en la pieza de PR respecto a la biopsia (6 cilindros). Y concluyen que aún el más pequeño de los focos en la biopsia, no garantiza la insignificancia del tumor en la pieza ${ }^{21}$.

En otras series, sólo el 53\% de los tumores potencialmente insignificantes en la biopsia se manifestaron como tal en la pieza de PR, por lo que concluyen que el valor predictivo de un solo 
foco de carcinoma de próstata bien diferenciado en la biopsia es limitado para predecir esto en la pieza de $\mathrm{PR}$, ya que tumores moderados e incluso avanzados fueron hallados en las piezas de PR. Creen que la localización multifocal de los tumores prostáticos el la razón más probable para la limitada predictibilidad de un solo cilindro en la biopsia ${ }^{22-24}$.

En otros trabajos, las conclusiones son semejantes. Un 30\% de correlación entre los tumores insignificantes en la biopsia, y el resultado final en la pieza de PR es el resultado de Bocón-Gibod y cols., que realizaron, al igual que nosotros biopsias de 10 cilindros ecodirigidas y consideran 1 solo cilindro y Gleason $\leq 6$ en la biopsia; y volumen $<0,5 \mathrm{ml}$ y Gleason $\leq 6$ en la pieza de PR. Y añaden que, aunque este grupo de pacientes, quizás hayan sido sobre-tratados, no es posible identificarlos pre-operatoriamente para adoptar con seguridad una actitud expectante. Pero otras series, solo encuentran un $16 \%$ de tumores verdaderamente insignificantes en la pieza de prostatectomía radical, pero afirman que es el número de cilindros positivos en la biopsia es uno de los factores predoctores de bajo volumen tumoral más importante $1,25-27,29$.

Sin embargo, en algunas series, incluida la nuestra, lo que sí se ha observado es una mayor correlación de estos tumores potencialmente insignificantes con tumores órgano-confinados en la pieza de PR. Los niveles de PSA, la densidad y los hallazgos en la biopsia son factores predictivos de la extensión del tumor, por lo que en casos seleccionados podría ser razonable el seguimiento de los potencialmente insignificantes con medidas seriadas del PSA y biopsias repetidas, que es lo que se denomina "seguimiento activo"18,27.

Nuestro trabajo, es una revisión y análisis retrospectivo de la serie de prostatectomías radicales realizadas en nuestro centro sobre pacientes con un tumor en la biopsia potencialmente insignificante. En la mayoría de los grupos, el tumor insignificante en la biopsia es definido como un solo cilindro positivo y un Gleason $\leq$ a 6 . Nosotros consideramos además un nivel sérico de PSA $\leq 10$ Así mismo, han definido el tumor insignificante en la pieza de prostatectomía radical como un volumen tumoral < 5\% y un grado de Gleason $\leq$ a 6 . Estos son los parámetros que nosotros hemos considerado para definir "insignificante" en cada uno de los grupos.
Aunque los resultados presentados de nuestra serie, y los descritos en la literatura sugieren fuertemente la posiblilidad de predecir tumores minimamente invasivos combinando niveles séricos de PSA y biopsia, aún no están definidos los algoritmos capaces de predecir con seguridad el cáncer insignificante.

Ademas, existen numeros estudios que concluyen que el infradiagnóstico de cáncer de próstata es aun muy frecuente, y superior al sobrediagnóstico, ya que ronda en algunas series el $25-30 \%$, por lo que la tendencia actual es bajar el nivel de PSA como límite inferior de la normalidad para realizar biopsia de prostática. Esto dificultaría aun más la posibilidad de realizar un tratamiento expectante, sobretodo en pacientes jóvenes, ya que por el momento, no contamos con los criterios clínicos suficientes para predecir los tumores realmente insignificantes ${ }^{28,30}$.

Una propuesta reciente del grupo de BocconGibod es la biopsia de saturación para re-evaluar aquéllos pacientes con tumores potencialmente insignificantes, ya que en su trabajo encontraron que un $70 \%$ de este grupo de pacientes tenian un tumor significante en la biopsia de saturación de 32 cilindros, y por lo tanto dejaban de ser considerados microfocales o insignificantes como se había concluído tras la primera biopsia y se les ofrecía un tratamiento activo ${ }^{29}$. La capacidad para decidir qúe tumores podrían ser tratados y cuáles manejados con una actitud expectante, requerirá largos estudios randomizados con un seguimiento suficientemente largo ${ }^{31}$.

Y sobretodo, aún desconocemos el punto de corte de PSA con elevada sensibilidad y especificidad simultáneas como para monitorizar a los varones sanos respecto al diagnóstico precoz del cáncer de próstata ${ }^{1,27,32-37}$.

\section{CONCLUSIÓN}

Con los resultados que hemos descrito, ya parece claro, que aún el más pequeño de los focos, no garantiza que el tumor vaya ser clínicamente insignificante. No sabemos, si una explicación podría ser la localización multifocal de los tumores en la próstata. Lo que sí podemos decir con lo que hemos observado, es que en general, los tumores microfocales en la biopsia tendrían un pronóstico clínico más favorable que le resto de los pacientes con mayor tumor en la biopsia. Por lo tanto, la posibilidad de predecir qué tumores podrían 
ser tratados de forma expectante, probablemente requiera estudios randomizados con períodos de seguimiento suficientemente largos. Nuestra serie igual que otras, muestra que no existe todavía un criterio clínico, biológico o patológico capaz de identificar en un individuo aquéllos tumores de próstata insignificantes que podrían ser susceptibles de tratamiento expectante.

Resumimos nuestras conclusiones en los siguientes puntos:

1. Los tumores de bajo riesgo, con baja carga tumoral en la biopsia, no siempre expresan tumores insignificantes. En nuestra serie, un $41^{\prime} 5 \%$ de los supuestamente insignificantes en la biopsia, lo eran finalmente, en la pieza de PR.

2. Aún el más pequeño de los focos en la biopsia, no garantiza tumor clínicamente insignificante.

3. Aún no podemos decir que existan algoritmos diagnósticos para identificar preoperatoriamente el teórico cáncer de próstata insignificante, como posibles candidatos a vigilancia expectante.

4. Un seguimiento exhaustivo de los niveles séricos de PSA, con una buena comunicación con el paciente, o la propuesta de una rebiopsia de saturación a este grupo de pacientes, podrían ser alternativas por el momento para llevar a cabo la vigialancia activa de este grupo de pacientes.

\section{REFERENCIAS}

1. Catalona WJ, Smith DS, Ornstein DK. Prostate cancer detection in men with serum PSA concentrations of 2.6 to 4.0 $\mathrm{ng} / \mathrm{ml}$ and benign prostate examination. Enhancement of specificity with free PSA measurements. JAMA. 1997;277(18): 1452-1455

2. Boccon-Gibod LM, Dumonceau O, Toublanc M, Ravery V, Boccon-Gibod LA. Micro-focal prostate cancer: a comparison of biopsy and radical prostatectomy specimen features. Eur Urol. 2005;48(6);895-899.

3. Alschibaja M, Wegner M, Massmann J, Funk A, Hartung R, Paul R. Prostate cancer volume - can it be predicted preoperatively? Urol International 2005;75(4):354-359.

4. Allan RW, Sanderson H, Epstein JI. Correlation of minute (0.5 MM or less) focus of prostate adenocarcinoma on needle biopsy with radical prostatectomy specimen: role of prostate specific antigen density. J Urol. 2003; 170(2 Pt 1):370-372.

5. Hoedemaeker RF, Van Der Kwast TH, Schroder FH. The clinical significance of a small focus of well-differentiated carcinoma at prostate biopsy. BJU. 2003;92 Suppl 2: 92-96.

6. Anast JW, Andriole GL, Bismar TA, Yan Y, Humphrey PA. Relating biopsy and clinical variables to radical prostatectomy findings: can insignificant and advanced prostate cancer be predicted in a screening population?. Urology. 2004;64(3):544-550.

7. Gardner TA, Lemer ML, Schlegel PN, Waldbaum RS, Darracott Vaughan E, Steckel J. Microfocal prostate cancer: biopsy cancer volume does not predict actual tumour volume. BJU. 1998;81 (6):839-843.
8. Ravery V, Szabo J, Toublanc M, Boccon-Gibod LA, Billebaud T, Hermieu JF, et al. A single positive prostate biopsy in six does not predict a low-volume prostate tumour. BJU. 1996;77(5): 724-728.

9. Kattan MW, Eastham JA, Wheeler TM, Maru N, Scardino PT, Erbersdobler A, et al. Counseling men with prostate cancer: a nomogram for predicting the presence of small, moderately differentiated, confined tumor. J Urol. 2003;170(5):1792-1797.

10. Miyake H, Sakai I, Harada K, Hara I, Eto H. Prediction of potentially insignificant prostate cancer in men undergoing radical prostatectomy for clinically organ-confined disease. Int $\mathrm{J}$ Urol. 2005; 12(3):270-274.

11. Klotz L. Active surveillance for favorable-risk prostate cancer: who, how and why?. Nat Clin Pract Oncology. 2007;4(12):692-698.

12. Noguchi M, Stamey TA, McNeal JE, Yemoto CM. Department of Urology, Stanford University School of Medicine, Stanford, California. Relationship between systematic biopsies and histological features of 222 radical prostatectomy specimens: lack of prediction of tumor significance for men with nonpalpable prostate cancer. J Urol. 2001;166(1):104-109.

13. Sebo TJ, Bock BJ, Cheville JC, Lohse C, Wollan P, Zinke H. The percent of cores positive for cancer in prostate needle biopsy specimens is strongly predictive of tumor stage and volume at radical prostatectomy. J Urol. 2000;163(1):174-178.

14. Epstein JI, Chan DW, Sokoll LJ, Walsh PC, Cox JL, Rittenhouse $\mathrm{H}$, et al. Nonpalpable stage T1c prostate cancer: prediction of insignificant disease using free/total prostate specific antigen levels and needle biopsy findings. J Urol. 1998; 160(6 Pt 2):2407-2411.

15. D’Amico AV, Ming-Hui Chen, Roehl KA, Catalona WJ. Identifying Patients at Risk for Significant Versus Clinically Insignificant Postoperative Prostate-Specific Antigen Failure. J Clin Oncol. 2005;23(22):4975-4979.

16. Jeldres C, Suardi N, Walz J, Hutterer GC, Ahvai S, Lattouf JB, et al. Validation of the contemporary Epstein Criteria for Insignificant Prostate Cancer in European Men. Eur Urol. 2007.

17. Bill-Axelson A, Holmberg L, Ruutu M, Aggman MH, Andersson SO, Brattel S, et al. Radical Prostatectomy versus Watchful Waiting in Early Prostate Cancer. J Urol. 2005;352(19):19771984

18. Thompson IM, Ankerst DP, Chi C, Lucia MS, Goodman PJ, Crowley JJ, Parnes HL, Coltman CA Jr. operating characteristics of Prostate-Specific Antigen in Men with an initial PSA level of $3.0 \mathrm{ng} / \mathrm{ml}$ or lower. JAMA. 2005; 66-71.

19. Wong YN, Mitra N, Hudes G, Localio R, Sanford Schvartz, J, Wan F, et al. Survival associated with treatment vs observation of localized prostate cancer in elderly men. JAMA. 2006; 296 (22):2683-2693.

20. Konety BR, Bird VY, Deorah S, Dahmoush L. Comparison of the incidence of latent prostate cancer detected at autopsy before and after the prostate specific antigen era. J Urol. 2005; 174(5):1785-1788.

21. Harlan SR, Cooperberg MR, Elkin EP, Lubeck DP, Meng MV, Mehta SS, et al. Time trends and characteristics of men choosing watchful waitinf for initial treatment of localized prostate cancer: results from capsure. J Urol. 2003;170(5):1804-1807.

22. Etzioni R, Penson DF, Legler JM, di Tomaso D, Boer R, Gann $\mathrm{PH}$, et al. Overdiagnosis due to Prostate-Specific Antigen screening: Lessons from US prostate cancer incidence trends. J Natl Cancer Inst. 2002;94(13):981-990.

23. Cheng L, Poulos CK, Pan C, Jones T, Daggy JK, Eble JN, et al. Preoperative prediction of small volume cancer (less than $0.5 \mathrm{ml}$ ) in radical prostatectomy specimens. J Urol. 2005; 174(3):898902 . 
24. Thompson IM, Pauler DK, Goodman PJ, Tangen CM, Lucia MS, Parnes HL, et al. Prevalence of prostate cancer among men with a prostate - specifec antigen level or $=4.0 \mathrm{ng} / \mathrm{ml}$. N Engl J Med. 2004;350(22):2239-2246.

25. Thompson KE, Kadee E, Hernández J, Canby-Hagino, Edith D, Troyer D, Thompson IM. Prognosis features in men who died of prostate cancer. J Urol. 2005; 174(2):553-556.

26. Walsh RM, Thompson IM. Prostate cancer screening and didease mamagemente: how screening may have an unintended effect on survival and mortality-the camel's nose effect. J Urol. 2007;177(4):1303-1306.

27. Bangma $\mathrm{CH}$, Roemeling S, Schroder FH. Overdiagnosis and overtreatment of early detected prostate cancer. J Urol. 2007; 25(1):3-9.

28. Graif T, Loeb S, Rochl KA, Gashti SN, Grffin C, Yu X, et al. Under diagnosis and over diagnosis of prostate cancer. J Urol. 2007; 178(1):88-92.

29. Boccon -Gibod LM, de Longchamps NB, Toublanc M, Boccon Gibod LA, Ravry V. prostate saturation biopsy in the reevaluation of microfocal prostate cancer. J Urol. 2006;176(3): 961-963.

30. Johnstone PA, Rossi PJ, Jani AB, Master V. Insignificant prostate cancer on biopsy: pathologic results from subsequent radical prostatectomy. Prostate Cancer Prostatic Dis. 2007; 10(3):237-241.

31. Samaratunga H, Yaxley J, Kerr K, McClymont K, Duffy D. Significance of Minute Focus of Adenocarcinoma on Prostate Needle Biopsy. J Urol. 2007;70(2):299-302.

32. Allan RW, Sanderson H, Epstein JI. Correlation of minute (0.5 MMor less) focus of prostate adenocarcinoma on needle biopsy with radical prostatectomy specimen: role of prostate specific antigen density. J Urol. 2003;170(2 Pt 1):370-372.
33. Taverna G, Colombo P, Seveso M, Giusiti G, Piccinelle A, et al. Single small focus of prostate adenocarcinoma $(<$ or $=1 \mathrm{~mm}$ and too small for grading) and clinical significant disease after radical prostatectomy. Arch Ital Urol Androl. 2006;78(2): 57-60.

34. Catalon WJ, Smitha DS, Ornstein DK. Prostae cancer detection in men with serum PSA concentrations of 2.6 to $4.0 \mathrm{ng} / \mathrm{ml}$ and benign prostate examination. Enhancement of specificity with free PSA measurements. JAMA. 1997;277(18):1452-1455.

35. Epstein JI, Walsh, PC, Carmichael M, Brendler CB. Pathologic and clinical findings to predict tumor extent of nonpalpable (Stage T1c) prostate cancer. JAMA. 1994;271(5):368-374.

36. Boccon Boccon-Gibod LM, de Longchamps NB, Toublanc M, Boccon-Gibod LA, Ravery V. Prostate saturation biopsy in the reevaluation of microfocal prostate cancer. J Urol. 2006;176 (3):961-963.

37. Ochiai A. Troncoso P. Chen ME. Lloreta J. Babaian RJ. The relationship between tumor volume and the number of positive cores in men undergoing multisite extended biopsy: implication for expectant management. J Urol. 2005;174(6):2164-2168.

Correspondencia autor: Dra. E. Romero Selas

Servicio de Urología.

Hospital Juan Canalejo

Xubias de Arriba, 84 - 15006 A Coruña

Tel: 981178000

E-mail autor: eromeroselas78@hotmail.com

Información artículo: Original - Cáncer de próstata

Trabajo recibido: enero 2008

Trabajo aceptado: febrero 2008 